\title{
Nano-Process Technology for Synthesis and Handling of Nanoparticles ${ }^{\dagger}$
}

\author{
F.E. Kruis and $\mathbf{H}$. Fissan \\ Gerhard-Mercator-Universität Duisburg, Germany*
}

\begin{abstract}
The term nanoparticle is used nowadays to indicate particles with diameters smaller than $100 \mathrm{~nm}$. The reduced size of nanoparticles is responsible for changed electronic, optical and magnetic properties of nanoparticles and nanostructured materials in comparison with the bulk material. This can be used for new applications such as quantum dots, luminescent materials, gas sensors, conductive films, and capacitive, resistive, magnetic and optical materials.

An overview is given of the different synthesis processes used to provide nanoparticles with the required properties for functional applications, especially electronic and optical ones. We will concentrate on gas-phase processes to show their advantages. Apart from the synthesis techniques, handling techniques are described. Some examples from our own research are given, in which the use of electrical forces plays a central role: size fractionation, deposition and mixing can all be controlled by using charged particles.
\end{abstract}

\section{Introduction}

Nanoparticles have attracted the attention of an increasing number of researchers from several disciplines in the last ten years. The term "nanoparticle" came into frequent use in the early 1990s together with the related concepts, "nanoscaled" or "nanosized" particle. Until then, the more general terms "submicron" and "ultrafine" particles were used. The term nanoparticle is generally used now in the materials science community to indicate particles with diameters smaller than $100 \mathrm{~nm}$.

Nanoparticles can be used to prepare nanophase materials, which are materials with a three-dimensional structure and a domain size smaller than 100 $\mathrm{nm}$. Nanophase materials are usually produced by compaction of a powder of nanoparticles. They are characterised by a large number of grain boundary interfaces in which the local atomic arrangements are different from those of the crystal lattice. "Nanocomposites" consist of nanoparticles dispersed in a continuous matrix, creating a compositional heterogeneity of the final structure. The nanocomposites usually involve a ceramic or polymeric matrix and are not restricted exclusively to thin films.

Most of the synthesis methods are based on the

\footnotetext{
* Process and Aerosol Measurement Technology, GerhardMercator-Universität Duisburg, D-47057 Duisburg, Germany

† Received: May 31, 1999
}

production of disperse materials systems, a domain of chemical engineering. This is also true for the conditioning processes, necessary for bringing the nanoparticles in a form which is suitable for application, which can be thought of as specialised unit operations. The experience and the special methods developed by chemical engineers with respect to dispersed materials systems are needed to understand, optimise, and scale-up the processes used for the synthesis and applications of nanoparticles. This field can be described as nano-process technology.

\section{Properties of nanoparticles}

The small size of nanoparticles, which is responsible for the different properties (electronic, optical, electrical, magnetic, chemical and mechanical) of nanoparticles and nanostructured materials with respect to the bulk material, makes them suitable for new applications. Of a size between the molecular and bulk solid-state structures, nanoparticles have hybrid properties which are incompletely understood today, creating a challenge for theoreticians as well. Some examples of these properties are lower melting temperature, increased solid-solid phase transition pressure, quantum effects, non-linear optical properties, lower effective Debye temperature, decreased ferroelectric phase transition temperature, higher self-diffusion coefficient, luminescence and catalytic activity [1] 


\section{Functional applications of nanoparticles}

Structural applications of nanoparticles are based on the mechanical properties of the nanophase materials, leading to, e.g., superplastic ceramics or extremely hard metals, using the nanoparticles rather as passive basic building units. Functional applications, however, rely on a transformation of external signals such as the filtering of incident light, the change of electrical resistance in different gas concentrations, and luminescent behaviour when electrically activated. Even before the 1990s, nanoscale catalysts and pigments were used, based mainly on the large surface area to volume ratio in these systems. After 1980, a renewed interest took place in nanomaterials research. Brus [2] suggested that quantum confinement effects occur specifically in semiconductor nanoparticles. Birringer et al. [3] developed a method for synthesising amounts of weakly agglomerated nanoparticles for producing nanophase materials.

In this paper, the use of nanoparticles for electronic and optical applications is discussed [1]. A broad spectrum of materials including insulators, semiconductors, superconductors, metals and alloys, optical active materials, and artificially structured materials can be used for these purposes. Most applications use films composed of nanoparticles, supported by a substrate material.

When electrons are confined to a small domain such as a nanoparticle, the system is called a "quantum dot" or zero-dimensional (0-D) structure. In this case, the electrons behave like "particles-in-a-box", and their resulting new energy levels are determined by quantum "confinement" effects. These new energy levels give rise to a modification of the optoelectronic properties as compared with the corresponding properties determined by the bulk material electronic structure [2]. Quantum confinement effects lead, for example, to higher energy level transitions as compared with the bulk material observed as a shorter wavelength optical absorption edge, indicated by a spectral "blue shift". Quantum dots can be also used to produce light emitters of various colours by "band gap tuning" using particle size effects. As an example, light-emitting diodes (LEDs), which have a voltagecontrolled, tuneable output colour, were produced [4].

Although these applications seem very attractive, the processes for producing quantum dot devices are technologically very demanding, as the nanoparticles should be of controlled size, monodisperse within a few percent, spherical, monocrystalline, and should have a stable and passivated surface. A current tech- nological approach to circumvent the inherent reactivity and instability of nanoparticles is to embed them in a glass or in a semiconductor which has a wider band gap. These nanocomposite structures can then be used in real-life optical and electrooptical applications. For semiconductor applications other than optical, the nanoparticles should be efficiently electrically contacted. Since attaching electrical leads to an array of quantum dots is a formidable technological task, one way of making an efficient contact is to use a semiconductor structure consisting of an ensemble of quantum dots. Increasing the contact area between nanoparticles and electrode to increase the signals is possible by depositing the nanoparticles on a very porous $\mathrm{TiO}_{2}$ electrode [5].

In microelectronics, the need for faster switching times calls for a reduction in the size of the electronic components. At present, one switching cycle in a transistor gives rise to the movement of many thousands of electrons, requiring a considerable amount of switching energy and producing excessive heat which can hardly be dissipated in high-frequency applications. This calls for the development of single-electron devices, in which the switching occurs with the motion of only a single electron. Such a device can be realised by a quantum dot in which the charge of an added electron effectively blocks the flow of other electrons by the so-called "Coulomb blockade". Both switching - which performs logic operations - and storage of information are possible with these devices. The problem here is also how to contact the nanoparticles electrically.

The interest in luminescent $\mathrm{Si}$ is due to the desire for the integration of optoelectronic devices, now based mainly on III-V semiconductors which are not yet integrable monolithically with the dominant Si microelectronics. Hence light-emitting Si would open the way for optical and electrical functionality on one integrated monolithic Si chip. $\mathrm{Si}$ in its bulk form is not luminescent but luminescence has been obtained from Si nanoparticles.

A gas sensor consists quite generally of a material with measurable physical properties, e.g. electrical or optical, which change when a gas is present. The simplest "nanomaterials"-based sensor concept involves measuring the changes in the electrical resistance of a nanocrystalline material when exposed to hydrogen. It has been shown that gas sensors based on nanoparticles are more sensitive than their bulk counterparts.

Nanometre particles have also found new applications in the production of resistors and varistors. Granular films consisting of small conducting particles embedded in an insulating matrix of ceramic or 
glass exhibit a great variability of the electrical conductivity by a proper control of the volume fraction [6]. They can be used as thick-film resistors. They were also shown to increase the breakdown voltage of varistors due to their large volume of grain boundaries.

Nanometre-structured, polycrystalline conducting thin films have also found potential in the production of transparent conductive electrodes for photoconductor and solar cell applications, where a large surface area is needed and epitaxial films are not feasible. By spraying a colloid of 3-nm nanoparticles on a heated substrate, Pehnt et al. [7] produced very smooth conducting thin CdTe films without gaps and with a roughness smaller than $5 \mathrm{~nm}$, for use in solar cells. Transparent conducting films based on nanoparticles, usually $\mathrm{SnO}_{2}$ and $\mathrm{In}_{2} \mathrm{O}_{3}$, have the advantage that they display minimal light absorption and scattering.

Dielectric elements based on nanometre structures are of recent interest due to the reduction in capacitance caused by the continuing scaling down of dynamic random access memories (DRAMs), which causes materials with ever larger dielectric permittivities to be sought. One method for increasing the dielectric permittivity is to disperse conductive particles in a dielectric matrix. Using nanoparticles keeps the dissipation factor low.

Other applications of nanoparticles include their use to improve high-temperature superconductors (HTS), thermoelectric materials, optical materials for attenuating UV radiation, electrochromic materials which can be used for smart windows and magnetics.

\section{Synthesis methods of nanoparticles}

At present, there are many synthesis routes which lead to nanoparticles, but they can be divided into three main groups: gas-phase synthesis, colloidal synthesis, and high-energy milling. Gas-phase processing systems have several inherent advantages:

- a very good control over size, shape and crystallinity

- a higher purity than in the case of liquid synthesis brought about by an improved control over the composition of the environment

- possibilities to synthesise multicomponent nanoparticles because of the convenient way in which the reactants are supplied and mixed

- cost advantages in comparison with - for instance vacuum synthesis techniques due to the use of atmospheric or low-pressure conditions

- easier control over the reaction rate and the reaction mechanism through temperature variation of the gas. Most synthesis routes presently in use are based on the production of condensable monomers. A supersaturation should therefore be achieved. This is made possible by physical or chemical methods. The physical methods involve some form of cooling of the vapour. Supersaturation can also be achieved by chemical reactions which produce a non-volatile condensable product. These reactions are usually decomposition reactions initiated by a rise in temperature.

Various heating and evaporation methods are possible. They are shown schematically in Figure 1. The

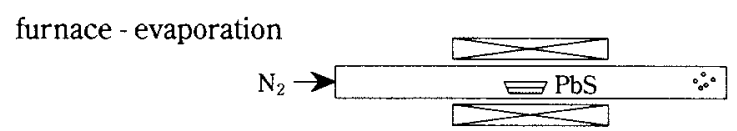

furnace - precursor pyrolysis

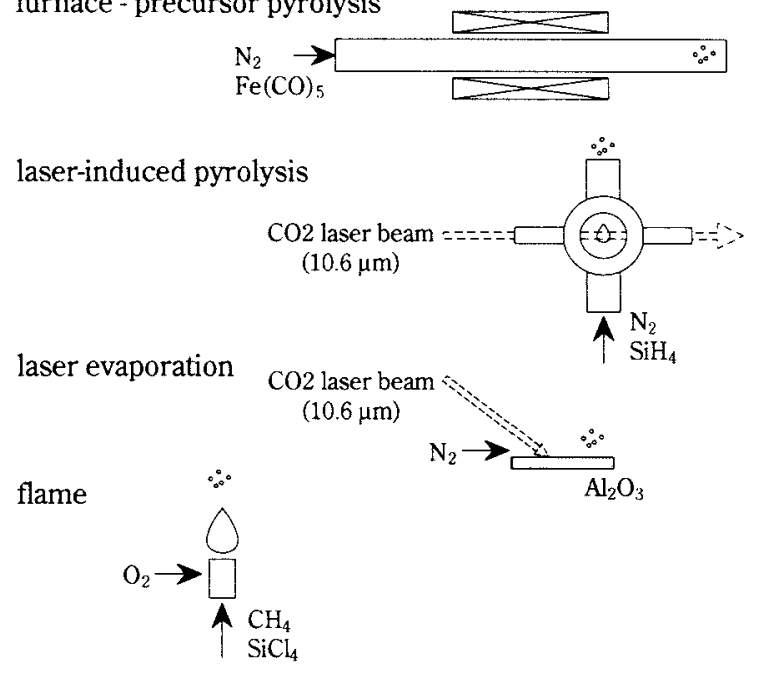

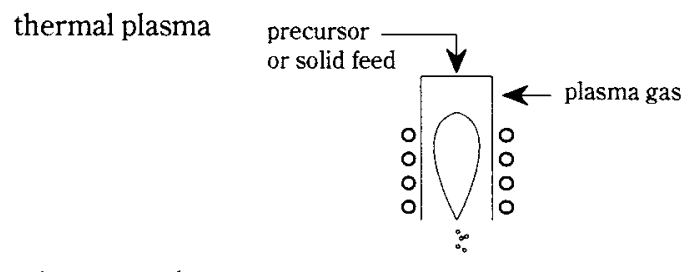

microwave plasma

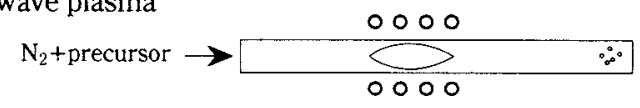

sputtering
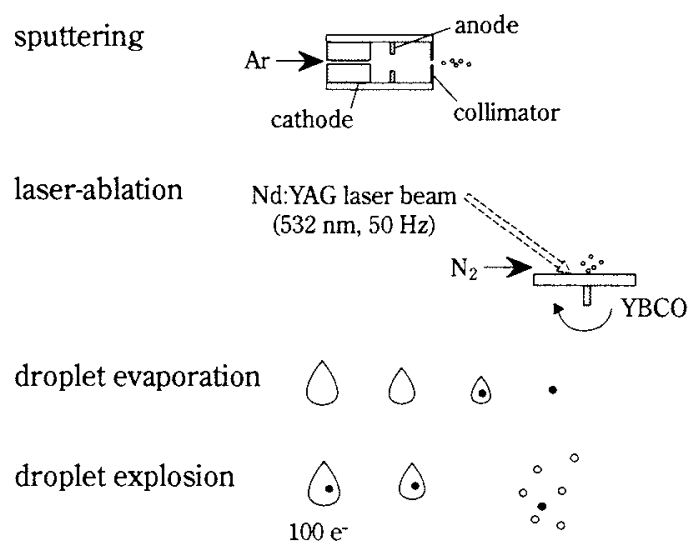

Fig. 1 Possible routes for synthesising nanoparticles 
simplest method is to heat a crucible containing the material in a furnace in a flowing inert gas. Nanoparticles are formed by subsequent cooling such as natural cooling or dilution cooling. Materials whose vapour pressure is too low to obtain appreciable particle density have to be fed into the furnace in the form of suitable precursors such as organometallics or metal carbonyls. These decompose in the oven to yield a condensable material.

In the laser pyrolysis technique, a flowing reactant gas is heated rapidly with an IR laser such as a cw $\mathrm{CO}_{2}$ laser. The source molecules are heated selectively by absorption of the laser beam energy. A gasphase decomposition of the reactants takes place due to the temperature increase, and supersaturation is created. As an example, $\mathrm{SiH}_{4}$ pyrolysis results in $\mathrm{Si}$ nanoparticle formation and $\mathrm{Fe}(\mathrm{CO})_{5}$ decomposition leads to $\mathrm{Fe}$ nanoparticles. The main advantage of laser-heating in gas-flow systems is the absence of heated walls, which reduces the danger of product contamination.

Laser vaporisation of solids uses a laser which evaporates locally a small area from a solid target in an inert gas-flow reactor. It is useful in the case of materials with a very high melting point and low vapour pressure such as refractory oxides.

Flame reactors employ the flame heat to initiate chemical reactions to produce condensable monomers. The flame route has the advantage of being an inexpensive method, however, it usually yields agglomerated particles and there is a risk of carbon contamination. Most flame reactors are operated at normal pressure, but they can also be operated at reduced pressures in order to decrease the particle size. Complex products such as nanocomposites can also be obtained.

A plasma reactor can also deliver the energy necessary to cause evaporation or initiate chemical reactions. The plasma temperatures are in the order of $10^{4} \mathrm{C}$, decomposing the reactants into ions and dissociating atoms and radicals. Solid powder feeds and droplets can also be decomposed by the plasma. Nanoparticles form upon cooling while exiting the plasma region. Complex materials such as multicomponent oxides, e.g. $\mathrm{BaFe}_{12} \mathrm{O}_{19}$, can be obtained by using appropriate combinations of solutions. Another method uses a microwave-generated plasma. The plasma enhances the kinetics of the chemical reactions leading to nanoparticle formation due to ionisation and dissociation of the reactive molecules. The charges on the particles might prevent further collisions and growth. A related method uses a high-current spark between two solid electrodes to evaporate the electrode material for creating nanoparticles. A plasma forms at the electrodes. This technique is used for materials with a high melting point such as Si or C.

Sputtering is a method of vaporising materials from a solid surface by bombardment with high-velocity ions of an inert gas, e.g. Ar or $\mathrm{Kr}$, causing an ejection of atoms and clusters. Sputter sources are normally used in vacuum systems, below $10^{-3}$ mbar. It was shown, however, that a $\mathrm{DC} / \mathrm{RF}$ magnetron sputter source can be operated in the mbar range, ejecting nanoparticles of sizes between 5 and $20 \mathrm{~nm}$. Sputtering has the advantage that it is mainly the target material which is heated and that the composition of the sputtered material is the same as that of the target.

Nanoparticle formation does not always rely on homogeneous nucleation from the gas phase. The ablation of a solid source with a pulsed laser can also yield nanoparticles, but the formation mechanism does not necessarily involve a homogeneous nucleation step. Instead, clusters or even larger particles are ejected from the surface which may undergo coagulation. One important characteristic of this method is that the nanoparticles have the same stoichiometry as the substrate, which is difficult to achieve for some materials using normal evaporation sources. A micron-sized aerosol droplet may also yield nanoparticles by evaporating a solute-containing droplet. By choosing a sufficiently dilute solute, nanoparticles consisting of the solid residue can be obtained. Electrospray systems are often applied as droplet generators, as they produce very small droplets which are quite monodisperse in comparison with other spray processes. Additionally, droplet explosion due to an electrical charge which exceeds the Rayleigh limit during evaporation may yield even smaller droplets. Finally, initiating homogeneous nucleation inside aerosol droplets can result in many nanosized nuclei in the droplet, which upon drying and ultimate fragmentation in the gas will yield nanoparticles. A more complete review of synthesis techniques can be found in [1].

\section{Methods to synthesise monosized nanoparticles}

Size control is of course necessary when size-dependent properties are sought. Gas-phase processes yield particles with a polydisperse size distribution, with standard deviations of 1.3 to 1.6 . When a narrow size distribution is required, the first process requirement is to make sure that all the particles experience the same time-temperature history. This method is used 


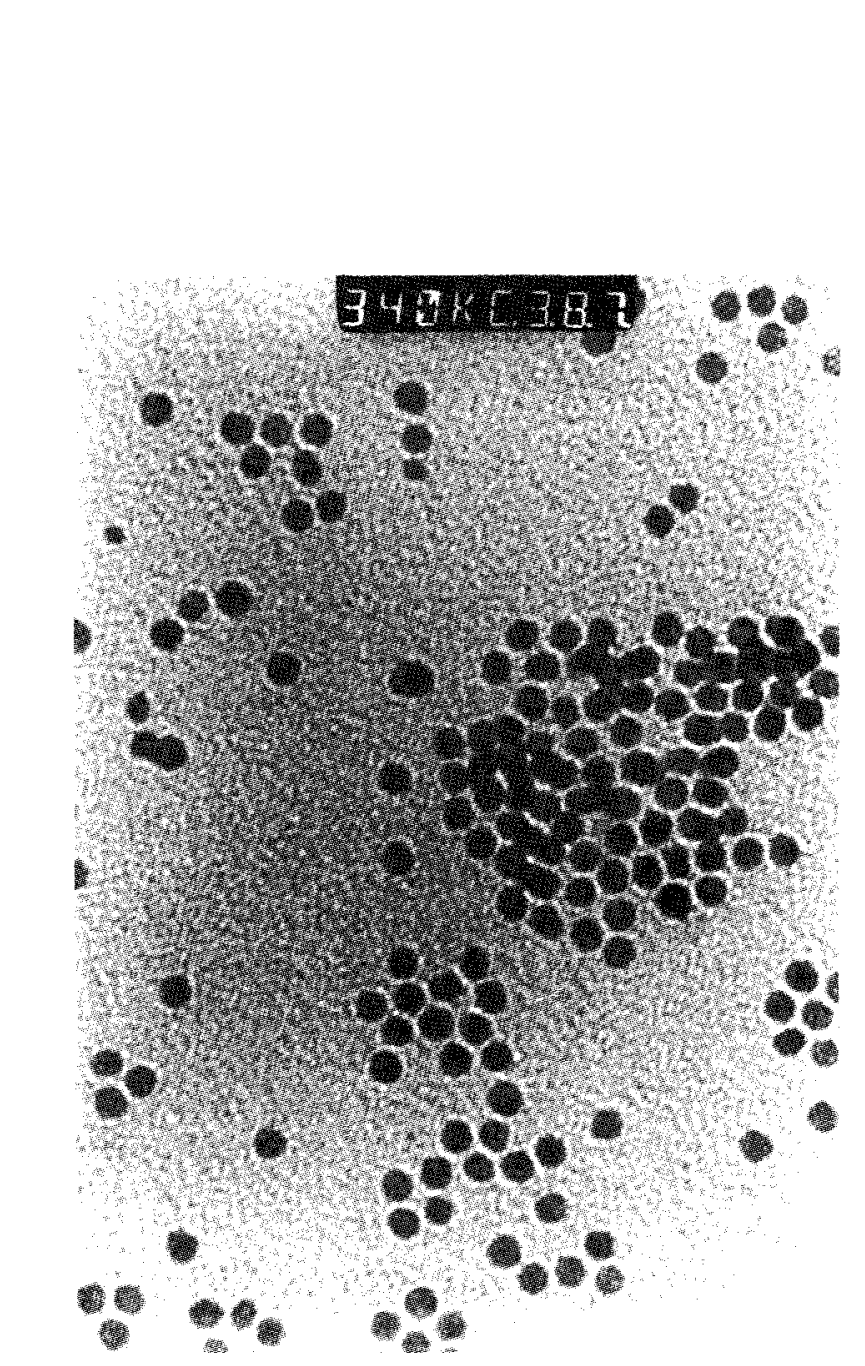

Fig. $2 \mathrm{Ag}$ nanoparticles with a mobility diameter of $10 \mathrm{~nm}$, obtained by size fractionation using a Differential Mobility Analyser.

in the so-called cluster machine [8] which is commercially available in Japan. When operated correctly, geometrical standard deviations of about $1.2-1.3$ can be obtained. Another method is to produce in a first step small nuclei and then to let them grow by controlled condensation [9]. This can yield still smaller standard deviations Monodisperse nanoparticles with standard deviations of around 1.1 can also be obtained by size classification techniques. The only technique currently being employed, apart from high-vacuum mass spectrometry, is the classification of singlecharged particles in an electric field. This so-called Differential Mobility Analysis (DMA) technique has been applied to produce monodisperse Ag (Figure 2), $\mathrm{Si}$ [10], Ga, GaAs, InP [11]and PbS [12] nanoparticles. Our work on the synthesis of $\mathrm{PbS}$ nanoparticles will be summarised in the remainder of this paper.

\subsection{Experimental technique for the synthesis of monodisperse $\mathrm{PbS}$ nanoparticles}

In the present work, a gas-phase synthesis method at normal pressure was developed for the preparation of sub-20 nm, crystalline, quasi-spherical, monodisperse $\mathrm{PbS}$ particles [12]. The experimental set-up is

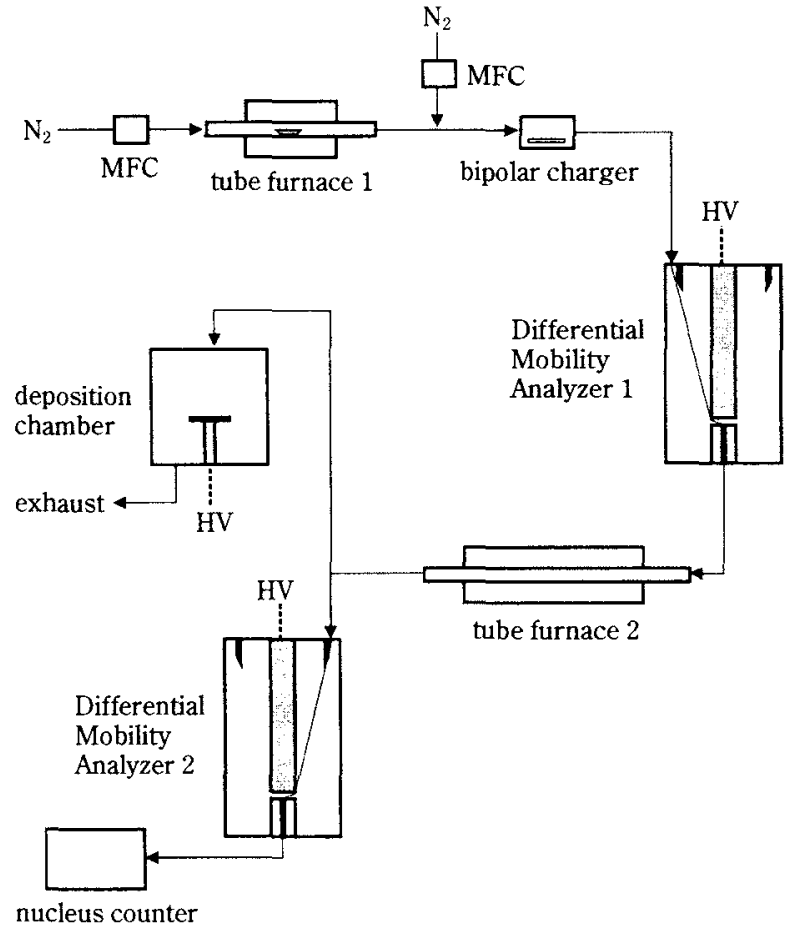

Fig. 3 Experimental set-up for synthesising monosized $\mathrm{PbS}$ nanoparticles.

depicted in Figure 3. It contains six elements: a nanocrystal source, an aerosol charger, a size classifier, a tube furnace, an electrostatic precipitator, and a particle size measurement system consisting of a Differential Mobility Analyser (DMA) and a condensation nucleus counter (3025, TSI, Minneapolis, USA). After an experimental comparison of four DMAs [13], a nanometre aerosol DMA (Nano-DMA) [14] was designed, optimised for the size range 3 to $50 \mathrm{~nm}$, see Figure 4.

$\mathrm{A} \mathrm{PbS}$ aerosol is formed by sublimating a $\mathrm{PbS}$ powder in a tube furnace and after dilution (1:2), charged by a radioactive source $\left(\mathrm{Kr}^{85}\right)$. Nitrogen obtained from evaporating liquid nitrogen is used as the carrier gas. The total gas flow was kept at $1.5 \mathrm{l} / \mathrm{min}$ by means of mass flow controllers (MFC), and the system was operated at atmospheric pressure. In a Differential Mobility Analyser, the charged particles are fractionated on the basis of their electric mobility, which is a function of their charge level, mass, and shape. The DMA (NANO-DMA, TSI, Minneapolis, USA) is capable of delivering monodisperse aerosols with sizes adjustable between 2 and $50 \mathrm{~nm}$ and standard deviations between 1.05 and 1.20 , depending on the flow conditions in the apparatus. In our studies, the flow ratio was chosen to provide a theoretical distribution in diameter of $\pm 0.1 D_{m}$. An electrostatic precipitator enables the aerosol to be deposited with almost $100 \%$ 
to meet the following conditions:

$$
T_{\text {high nucleation rate }}<T_{\text {furnace for sublimation }}<T_{\text {stoichiometric evaporation }} \text { (1) }
$$

and

$$
T_{\text {appreciable sintering }}<T_{\text {furnace for sintering }}<T_{\text {appreciable evaporation }}
$$

Fulfilling the first condition ensures that a sufficient number of particles is formed by homogeneous nucleation at temperatures lower than the temperature where off-stoichiometric evaporation starts. For InP and GaAs e.g., this relation is not fulfilled [15]. The second relation is clear from the earlier description of the experiments: crystalline and quasi-spherical particles have to be obtained by sintering at temperatures which do not cause extensive evaporation. For $\mathrm{PbS}$, there is for each particle size a temperature range of some $50 \mathrm{~K}$ where the sintering rate is appreciable, while at the same time the evaporation rate is such that the particles shrink but do not disappear. The optimum sintering temperature turns out to be $400^{\circ} \mathrm{C}$ for particles which have a mobility equivalent diameter of $15 \mathrm{~nm}$ and $500^{\circ} \mathrm{C}$ for particles which have a mobility equivalent diameter of $50 \mathrm{~nm}$.

\section{Handling methods of nanoparticles in the gas phase}

Nanoparticles leaving the reactor are not always suited to be directly integrated into their application. There are different conditioning steps possible which are indicated schematically in Figure 7.

Decreasing the degree of agglomeration is essential when nanoparticles have to be synthesised and when the formation of hard agglomerates has to be avoided. These agglomerates are formed by partial sintering of coagulated particles, forming material bridges which cannot be broken or dissolved. The most simple method is to dilute rapidly with a cool gas. One of the most successful cooling methods is the so-called inert-gas condensation, in which the nanoparticles are collected on a liquid $\mathrm{N}_{2}$ cooled surface (a "cold finger") and continuously scraped off in a funnel. The powder can be compacted in the reactor, which avoids exposure to air and oxidation. This method has been scaled up and is already being applied in industry, yielding up to 40 tons/month. Expansion of a condensable gas through a nozzle leads to very high cooling rates. Special nozzle design can result in a one-dimensional temperature gradient. This leads to a highly uniform quench rate and thus to nanoparticles with a narrow size distribution.

Chemical and physical transformations are applied in order to obtain the desired product. In some cases,
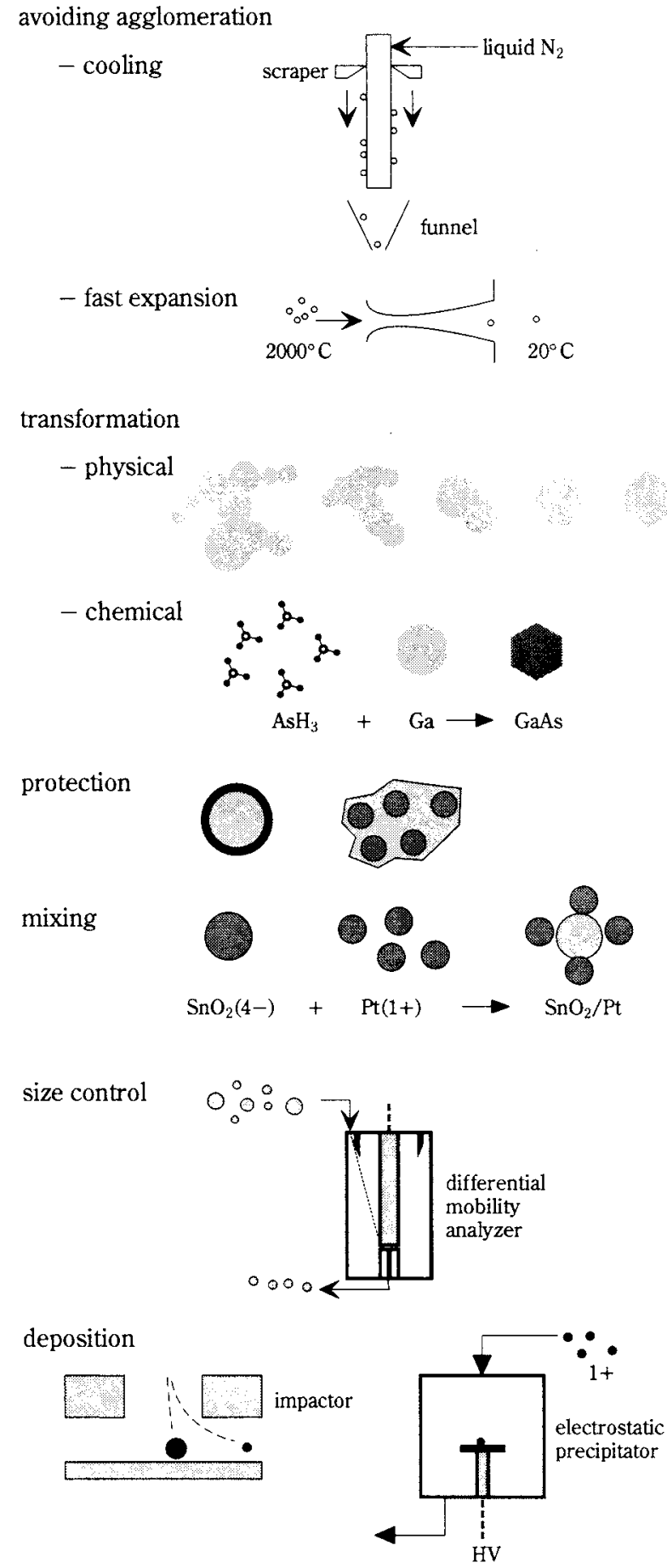

Fig. 7 Possible handling methods for nanoparticles.

chemical reactions are used in order. As an example, in the case of GaAs synthesis, direct sublimation is not possible due to incongruent evaporation. Therefore $\mathrm{Ga}$ nanoparticles are synthesised by evaporation in a first furnace and then reacted in a second furnace with $\mathrm{AsH}_{3}$, resulting in GaAs nanoparticles [11]. Apart from chemical composition, the crystallinity is also important. Usually crystalline particles are required. In our $\mathrm{PbS}$ nanoparticle synthesis work [12], the as- 
formed nanoparticles are amorphous and not spherical and have to be sintered in order to obtain monocrystalline and spherical particles.

Protection of nanoparticles is important as they are very reactive due to their large surface area. This is the main handling problem. In order to protect them for oxidation, they can be embedded, e.g. in a polymer or glassy host, handled in an inert atmosphere or protected by a thin coating. As an example, a method to avoid oxidation of flame-generated nanoparticles is to encapsulate them in the reactor within a material, e.g. a protective layer of $\mathrm{NaCl}$, which can be removed later by washing or sublimation.

Nanocomposites demand a mixing of the components on the nano-scale. Usually this is no longer possible when the components are separately collected as powder, due to the presence of big aggregates. A mixing during the reaction or shortly afterwards, when the nanoparticles are still unaggregated or only slightly aggregated, is necessary to obtain a homogeneous mixing. A controlled mixing can be obtained by electrical bipolar mixing, in which two aerosols of different chemical composition are separately charged with opposite polarities and then mixed. The aggregation rate of particles of the same component is thereby slowed down, while the aggregation rate of particles of unequal components increases. This is shown in Figure 8, where this selectivity is shown as a function of particle size for singly charged particles. It can be seen that the two unequal charged particles have a 15-times-higher probability to aggregate than two equal charged particles. An example is the bipolar mixing of $\mathrm{BaZrO}_{3}$ with $\mathrm{YBa}_{2} \mathrm{Cu}_{3} \mathrm{O}_{7-\mathrm{x}}$ [16], in which it was possible to control the number of $\mathrm{BaZrO}_{3}$ particles combining with one $\mathrm{YBa}_{2} \mathrm{Cu}_{3} \mathrm{O}_{7-x}$ particles by the charge level on the $\mathrm{BaZrO}_{3}$ particles.

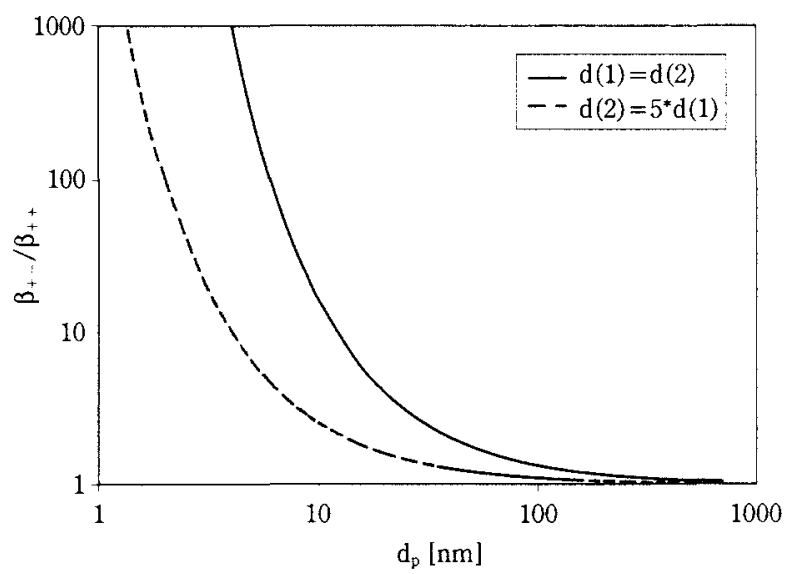

Fig. 8 Selectivity for aggregation of unequal components: ratio of collision rate of unequal charged particles and collision rate of equal charged particles (for singly charged particles)

\section{Deposition of nanoparticles}

After the synthesis, the particles have to be collected as a powder or brought onto a substrate. Powder collection can be accomplished by filtration, electrostatic precipitation or thermophoresis (the net movement of particles towards regions with a lower temperature) onto a cold surface. When thin or thick films of nanoparticles are required on a substrate, the usual way is to insert the substrate into the aerosol flow. The nanoparticles, which are often aggregated in micron-sized particles, will deposit due to the combination of thermophoresis and impaction due to inertia. A large part of the aerosol will, however, pass by. A higher deposition efficiency can be obtained by unipolarly charging the particles, and depositing them on a substrate placed on the central electrode in an electrostatic precipitator. A new type of unipolar charger we developed is shown in Figure 9 [17], which can be called a twin-Hewitt charger. Here, two corona discharge zones are connected by a charging zone. The aerosol flow is parallel to the corona wires and the wire mesh. The first main advantage of this new charger is that due to the use of a square-wave voltage, ions are drawn into the charging zone alternately from each corona discharging zone. This increases the mean ion concentration considerably, resulting in a higher $N t$-product. As a second advantage, the relatively low residence time in the inlet and outlet regions (some 10-20\% of the total residence time) should be mentioned. This is especially important for nanoparticles with potentially high diffusional losses.

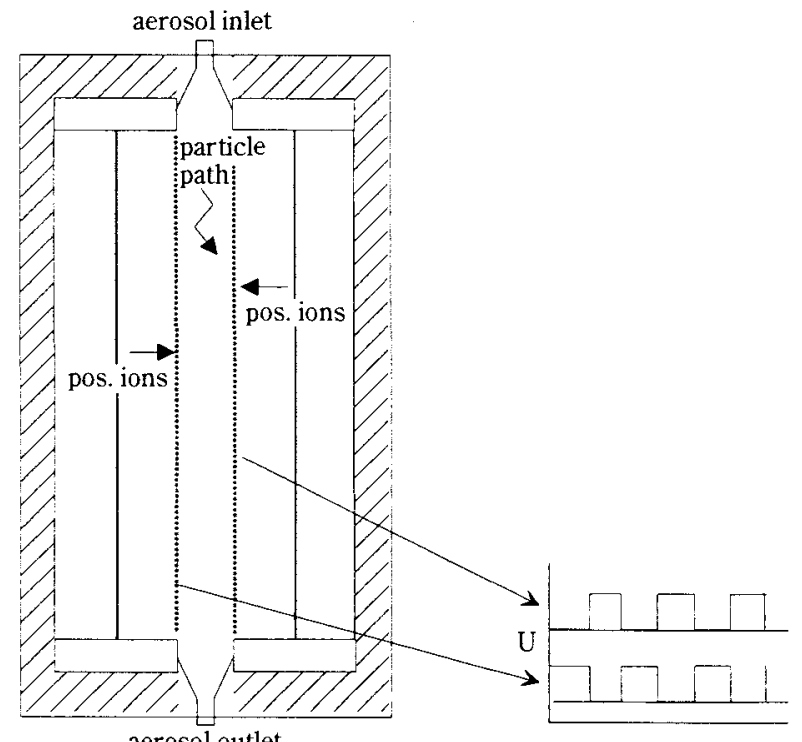

Fig. 9 Schematic design of the new unipolar charger for nanoparticles. 


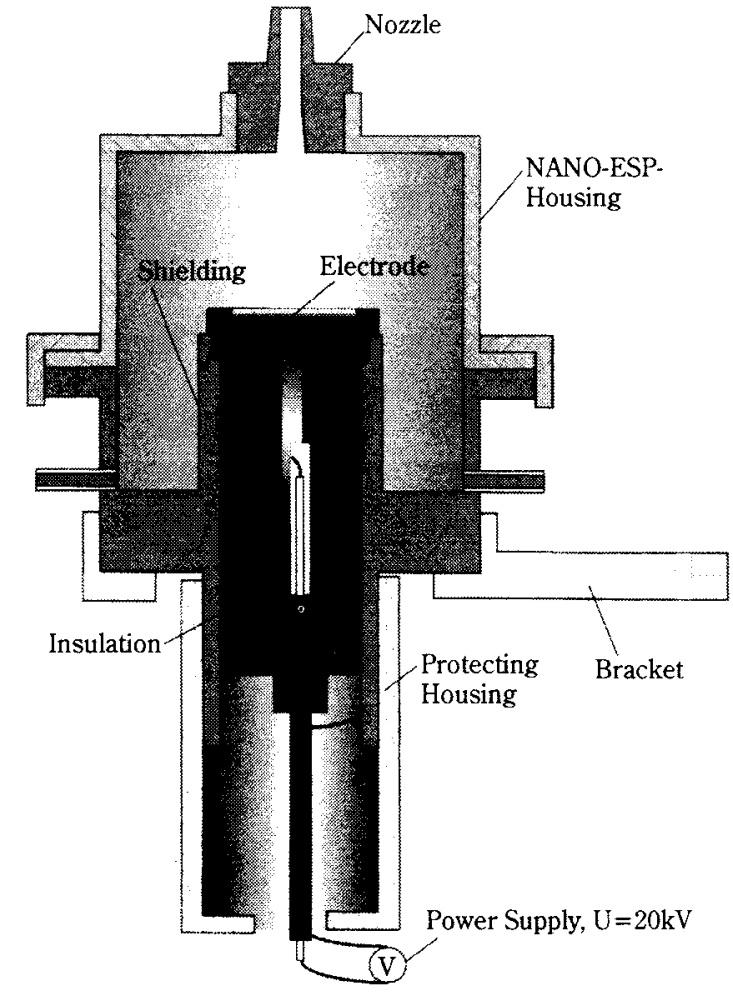

Fig. 10 Schematic design of the electrostatic precipitator.

Deposition of charged particles is achieved most efficiently by means of an electrostatic precipitator, which was specially developed [18] and is shown in Figure 10. Requirements for the ESP-design were almost complete particle sampling on a flat substrate and at least nearly homogeneous particle distribution on the substrate. To achieve better control of the particle deposition process, the charging and the deposition processes were separated. In the centre of the aerosol flow, the particle concentration distribution is homogeneous. If we put a round plate into the aerosol flow, the particles spread out because the flow goes around the obstacle. This affects the deposition pattern. On the other hand, an electric field with converging field lines on the plate competes with the flow-field effect on the particles. Thus both mechanisms may be used to achieve complete sampling on the one side and a homogeneous distribution on the other side. The voltage is applied between the central electrode and the walls of the electrically earthed expansion chamber. The design was guided by a complete numerical model. Particles up to $220 \mathrm{~nm}$ in diameter carrying one elementary charge can be sampled with the ESP with $100 \%$ efficiency.

Controlling the deposition pattern of nanoparticles on surfaces is another step towards the use of nanoparticles in functional applications. Nanoparticles were deposited in an electrostatic precipitator on microelectronic substrates with metal electrodes of micron-sized spacing. Control over particle deposition was obtained by creating a lateral electric field between the electrodes, resulting in the particles depositing preferentially on one electrode. Another method uses photoresist patterns on the substrate. The particles deposit preferentially on the areas without photoresist, probably due to charging of the photoresist. The photoresist is removed afterwards without visible loss of particles. As a first device application, metal-semiconductor-metal photodetectors with multifinger interdigitated electrodes were produced. After nanoparticle deposition, the detectors show a higher sensitivity, $4 \mathrm{x}$ on a GaAs substrate and $60 \mathrm{x}$ on a Si substrate [19].

\section{Conclusions}

Without doubt, nanoparticles show interesting properties of practical importance. We have listed some of the possible applications in the field of electronics and (electro)-optics. Many types of gaseous synthesis techniques exist. Essential for applications involving nanoparticles is the use of suitable handling techniques. Chemical engineering methods concerning dispersed materials systems are needed to understand, optimise and scale-up the processes used for the synthesis and applications of nanoparticles. This field can be described as nano-process technology. We have shown here some examples of handling techniques based on the use of electrical forces, which allows the use of monodisperse nanoparticles, controlled mixing of different nanoparticle components, and deposition control.

\section{References}

1) Kruis, F.E., H. Fissan and A. Peled: J.Aerosol Sci., 29 511 (1998)

2) Brus, L.E.: J.Chem.Phys. 795566 (1983)

3) Birringer, R., H. Gleiter, H.P. Klein, and P. Marquardt: Phys.Lett 102A 365 (1984)

4) Colvin, V.L., M.C. Schlamp, and A.P. Alivisatos: Nature 370354 (1994)

5) Weller, H.: Angew.Chem.Int.Ed.Engl. 3241 (1993)

6) Abeles, B., H.L. Pinch and J.L. Gittleman: Phys.Rev.Lett. 35247 (1975)

7) Pehnt, M., D.L Schulz, C.J. Curtis, K.M. Jones and D.S. Ginley: Appl.Phys.Lett. 72176 (1995)

8) Hayashi, C.: Mat. Sci. Forum 246 153(1997)

9) Bowles, R.S., J.J. Kolstad, J.M. Calo and R.P. Andres: Surf. Sci 106117 (1981)

10) Camata, R.P., H.A. Atwater, KJ. Vahala and R.C. Flagan: 
Appl.Phys.Lett. 683162 (1996)

11) Deppert, K., J.-O. Bovin, J.-O. Malm and L. Samuelson: J.Crystal Growth 16913 (1996)

12) Kruis, F.E., K. Nielsch, H. Fissan, B. Rellinghaus and E.F. Wassermann: App1.Phys.Lett. 73547 (1998)

13) Fissan, H., D. Hummes, F. Stratmann, P. Büscher, S. Neumann, D.Y.H. Pui and D.R. Chen: Aerosol Sci. Technol. 241 (1996)

14) Chen, D.R., D.Y.H., Pui, D. Hummes, H. Fissan, F.R. Quant, and G.J. Sem: J. Aerosol Sci. 29 497(1998)

15) Deppert, K, K. Nielsch, M.H. Magnusson, G. Dumpich, E. Kruis and H. Fissan: Nanostruct. Matls. 10565 (1998)
16) Takao, Y., M. Awano, Y. Kuwahara and Y. Murase.: Sensors and Actuators B 31131 (1996)

17) Kruis, F.E., F. Otten, F. Jordan and H. Fissan: J. Aerosol Sci. 29 S1021 (1998)

18) Dixkens, J. and H. Fissan. Design of a sampling system for off-line particle analysis, pp. 214-222 in Fine Solid Particles (ed. J. Schwedes, S. Bernotat), Shaker Verlag, Aachen, Germany (1997)

19) Prost, W., F.E. Kruis, F. Otten, K. Nielsch, B. Rellinghaus, U. Auer, A. Peled, E.F. Wassermann, H. Fissan and F.J. Tegude: J. Microelect. Eng. 41/42 535 (1998)

\section{Author's short biography}

\section{F.E. Kruis}

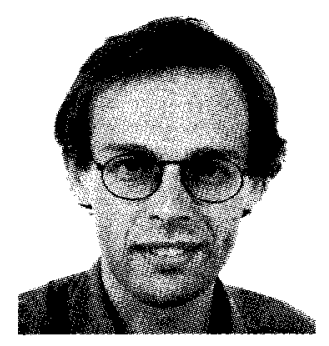

Einar Kruis graduated in Chemical Engineering from Delft University of Technology in 1988. In 1993 he completed his Ph.D. research on the laser-assisted synthesis of ultrafine ceramic particles, performed in the Particle Technology Group of Prof. B. Scarlett and the inorganic Chemistry Group of Prof. J. Schoonman. During this time he also worked with Prof. S. Pratsinis in Cincinnati, USA. He pursued his academic carrier from 1993 to 1995 in the Reactor Engineering Institute of the late Prof. J. Villermaux at the ENSIC in Nancy, France as post-doctoral fellow in the Human Capital and Mobility program of the EC.

Since 1995 he is an assistant professor in the Institute for Process- and Aerosol Measurement Technology at the Gerhard Mercator University in Duisburg, Germany. He currently works on synthesis and handling techniques of nanoparticles.

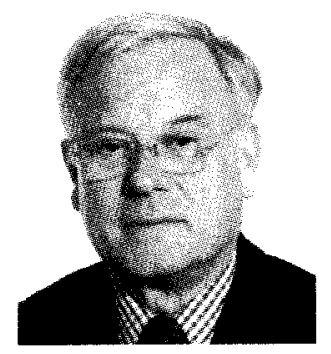

\section{H. Fissan}

Heinz Fissan is Professor at the Electrical Engineering Department and Director of the Institute for Process- and Aerosol Measurement Technology at the Gerhard Mercator University in Duisburg, Germany. His research deals with aerosols and their application in different fields of technology. Fields covered are aerosol measurement technology, gas filtration, atmospheric aerosols, clean technology and nanostructured materials. The last field includes the synthesis of nanostructures, the in-situ and off-line analysis of nanoparticles and nanostructures, the simulation of structure development and the application of nanoparticles and nanostructures in sensors and electronic and opto-electronic devices.

$\mathrm{He}$ is author/co-author of approximately 200 publications on these subjects. He is a fellow of the International Aerosol Research Assembly since 1990 and obtained the Max Planck Research Award in 1993. In 1998 he received the David Sinclair Award from the American Association for Aerosol Research (AAAR). 